ARTICLE

Received 14 May 2015 | Accepted 12 Dec 2015 | Published 25 Jan 2016

DOI: $10.1038 /$ ncomms10462

OPEN

\title{
Confinement-deconfinement transition due to spontaneous symmetry breaking in quantum Hall bilayers
}

D.I. Pikulin $1,2,3$, P.G. Silvestrov 4 \& T. Hyart ${ }^{3,5}$

\begin{abstract}
Band-inverted electron-hole bilayers support quantum spin Hall insulator and exciton condensate phases. Interest in quantum spin Hall effect in these systems has recently put them in the spotlight. We investigate such a bilayer in an external magnetic field. We show that the interlayer correlations lead to formation of a helical quantum Hall exciton condensate state. Existence of the counterpropagating edge modes in this system results in formation of a ground state spin-texture not supporting gapless single-particle excitations. The charged edge excitations in a sufficiently narrow Hall bar are confined: a charge on one of the edges always gives rise to an opposite charge on the other edge. Magnetic field and gate voltages allow the control of a confinement-deconfinement transition of charged edge excitations, which can be probed with nonlocal conductance. Confinement-deconfinement transitions are of great interest, not least because of their possible significance in shedding light on the confinement problem of quarks.
\end{abstract}

\footnotetext{
${ }^{1}$ Department of Physics and Astronomy, University of British Columbia, Vancouver, British Columbia, Canada V6T 1Z1. ${ }^{2}$ Quantum Matter Institute, University of British Columbia, Vancouver, British Columbia, Canada V6T 1Z4. ${ }^{3}$ Instituut-Lorentz, Universiteit Leiden, P.O. Box 9506,2300 RA Leiden, The Netherlands. ${ }^{4}$ Institute for Mathematical Physics, TU Braunschweig, 38106 Braunschweig, Germany. ${ }^{5}$ Department of Physics and Nanoscience Center, P.O. Box 35 (YFL), Fl-40014, University of Jyväskylä, Finland. Correspondence and requests for materials should be addressed to D.I.P. (email: pikulin@phas.ubc.ca).
} 
T he role of the electron-electron interactions for the experimentally accessible topological media is best appreciated in quantum Hall $(\mathrm{QH})$ systems. The fractionally charged quasiparticles have been studied at the fractional filling factors ${ }^{1,2}$, and the non-abelian excitations of more exotic QH states may eventually lead to a revolution in quantum computing ${ }^{3-7}$. However, Coulomb interactions play a crucial role also in the case of the integer filling factors ${ }^{1,8-13}$. Remarkably, interactions create a $\mathrm{QH}$ ferromagnetic ground state at total filling fraction $v=1$ even in the absence of Zeeman energy. In such systems, the $S U(2)$ spin rotation symmetry is spontaneously broken, resulting in the low-energy excitations being spin waves and charged topological spin textures, skyrmions $s^{1,8,12,13}$. The presence of a small symmetry-breaking Zeeman field does not change the low-energy excitations qualitatively.

In QH bilayer systems the role of spin is played by the layer index (pseudospin) ${ }^{8,10,11,13}$. In this case the $S U(2)$ pseudospin rotation symmetry is explicitly broken by the interactions, as they are larger within the layers than between the layers. The interactions favour the pseudospin orientations in $(x, y)$-plane, where the direction is chosen spontaneously (spontaneous $U(1)$ symmetry breaking) so that the $\mathrm{QH}$ bilayers realize an easy-plane ferromagnet. Since the spontaneously chosen direction in the $(x, y)$-plane corresponds to a spontaneous interlayer phase coherence, this easy-plane ferromagnetic state is equivalent to an exciton condensate 11,13 .

The QH ferromagnet and QH exciton condensate in electronelectron bilayers support a single chiral edge mode. However, the two Landau levels may also support counterpropagating edge modes. The natural hosts of such kind of $\mathrm{QH}$ states are systems supporting quantum spin Hall (QSH) effect ${ }^{14-19}$ due to inverted electron-hole bandstructure. In these materials the magnetic field allows tuning through the Landau level crossing ${ }^{20,21}$, where we expect to find a $\mathrm{QH}$ state with spontaneously broken (pseudo)spin-rotation symmetry. Thus, we argue that there exist four different experimentally accessible pseudospin ferromagnetic states, determined by spontaneously broken symmetry (SU(2) in single layer and $\mathrm{U}(1)$ in bilayer systems) and the edge structure (chiral or helical). All these possibilities are illustrated in Fig. 1.
In this paper, we concentrate on the helical $\mathrm{QH}$ exciton condensate state (broken $\mathrm{U}(1)$ symmetry and helical edge structure). Remarkably, we find that in this system the charged edge excitations in a sufficiently narrow Hall bar are confined: a charge on one of the edges is always connected to the opposite charge on the other edge through the bulk by a stripe of rotated pseudospins, and thus low-energy isolated charged excitations cannot be observed. The gapless single-particle excitations are prohibited since the electron-electron interactions lead to an edge reconstruction and opening of a single-particle gap ${ }^{22,23}$. However, unlike it happens in the existing examples, the helical exciton condensate creates long-range correlations between edges. We show that a magnetic field and gate voltages can be used to tune in and out of the exciton condensate phase. Thus this system provides a unique opportunity to study a confinementdeconfinement transition, similar to the one which is hypothesized to liberate the quarks from their color confinement at extremely high temperatures or densities ${ }^{24}$. Finally, we show that the confined and deconfined phases can be distinguished using non-local conductance.

\section{Results}

Helical quantum Hall exciton condensate phase. We consider bilayer QSH systems, such as InAs/GaSb ${ }^{17-19}$, described by the BHZ Hamiltonian ${ }^{15,17}$ (Supplementary Note 1). The important property of these systems is that there is a crossing of electron and hole Landau levels as a function of magnetic field at $B=B_{\text {cross }}$ (refs 20,21; Fig. 2a and Supplementary Fig. 1), where the band inversion is removed. Near this crossing the single-particle Hamiltonian is (Supplementary Note 1):

$$
\hat{H}_{0}=\sum_{k}\left[\hat{\psi}_{k, \uparrow}^{\dagger} \hat{\psi}_{k, \uparrow}-\hat{\psi}_{k, \downarrow}^{\dagger} \hat{\psi}_{k, \downarrow}\right] E_{G}\left(k l_{B}^{2}\right)
$$

where $E_{G}(y)=E_{G}\left(k l_{B}^{2}\right)$ is the energy-momentum dispersion of Landau levels and $\hat{\psi}_{k \uparrow(\downarrow)}^{\dagger}$ are the electron creation operators for the lowest electron and hole Landau levels. Here we have fixed the total filling factor of the Landau levels $v_{T}=v_{\uparrow}+v_{\downarrow}=1$, and used the fact that the momentum $k$ in the Landau level wavefunctions is directly connected to the position $y$ in the real space. Importantly, the spin and layer degrees of freedom are locked
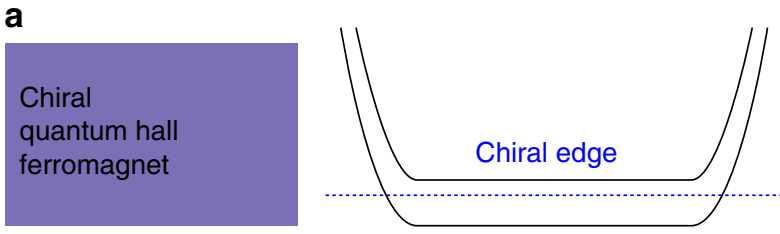

SU (2) symmetric interactions (Heisenberg ferromagnet)

\section{C}

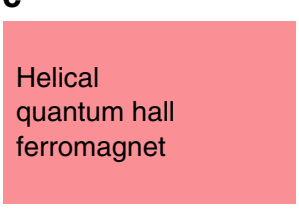

b

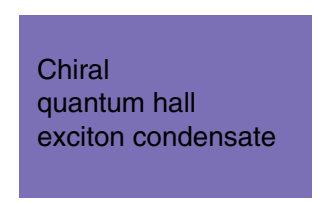

$U$ (1) symmetric interactions (easy-plane ferromagnet)

\section{d}
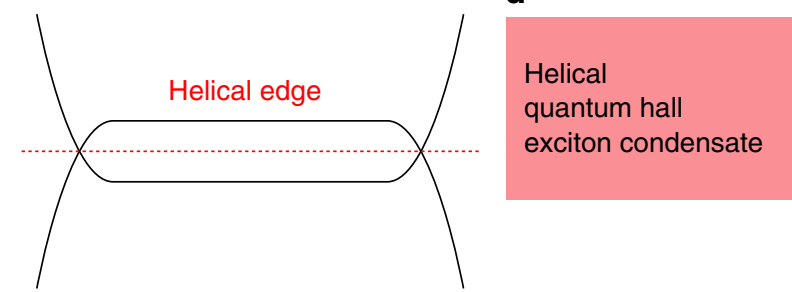

Figure 1 | Four different types of QH pseudospin ferromagnetic states at $\mathbf{v}=\mathbf{1}$. The single layer realizations ( $\mathbf{a}, \mathbf{c})$ realize Heisenberg ferromagnets because the interactions have SU(2) symmetry. The bilayer QH exciton condensates (b,d) can be described as an easy-plane ferromagnet with a spontaneously broken U(1)-symmetry. We argue that the classification is additionally enriched as the QH systems can support either chiral (a,b) or helical $(\mathbf{c}, \mathbf{d})$ edge excitations 
a

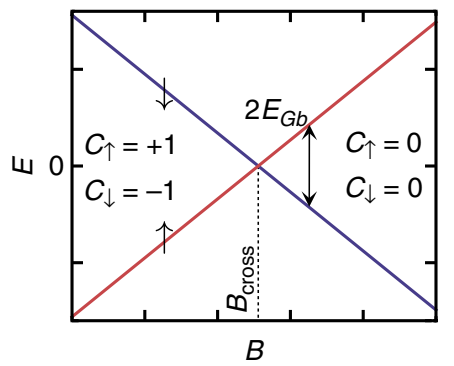

b $B<B_{\text {cross }}$

$E \uparrow$ Lower layer, spin down

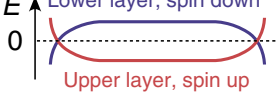

$\overrightarrow{y \equiv k l_{B}^{2}}$

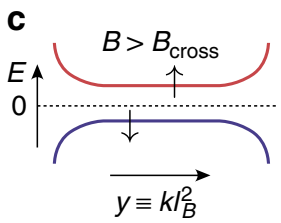

Figure 2 | Magnetic field and momentum dependence of the lowest Landau level energies. (a) There exists a robust crossing of the lowest Landau level energies as a function of magnetic field at $B=B_{\text {cross, }}$ because the energy of the electron-like Landau level with spin up (red line) increases and the energy of the hole Landau level with spin down (blue line) decreases as a function of $B$. We denote the energy separation between these two levels as $2 E_{\mathrm{Gb}}$. $(\mathbf{b}, \mathbf{c})$ Momentum (or equivalently position) dependencies of the lowest Landau level energies. The electron-like (hole-like) Landau level bends upwards (downwards) in energy near the edge of the sample. (b) For $B<B_{\text {cross }}$ the system supports helical edge modes protected by spin-resolved Chern numbers $C_{\uparrow}=-C_{\downarrow}=1$. (c) For $B>B_{\text {cross }}$ the edge is gapped according to the non-interacting theory $\left(C_{\uparrow}=C_{\downarrow}=0\right)$.

with each other, so that the pseudospin $\uparrow(\downarrow)$ means simultaneously up (down) spin and upper (lower) layer. The Fermi level is set to be at zero energy.

In the bulk the energy $E_{G}(y)=E_{\mathrm{Gb}}$ is independent of the momentum $\left(E_{\mathrm{Gb}}<0\right.$ for $B<B_{\text {cross }}$ and $E_{\mathrm{Gb}}>0$ for $\left.B>B_{\text {cross }}\right)$. When approaching the edge the Landau level originating from the electron (hole) band always disperses upwards (downwards) in energy. The spatial variation of $E_{G}(y)$ occurs within a characteristic length scale $l_{0} l_{B}$, which depends on the details of the edge, but due to topological reasons $E_{G}(y)>0$ reaches extremely large values (on the order of the energy separation between the bulk Landau levels) close to the edge (Supplementary Note 1). Therefore, for the magnetic fields $B<B_{\text {cross }}, E_{G}(y)$ goes through zero near the edge, yielding to the helical edge states (Fig. 2b). On the other hand for $B>B_{\text {cross }}$ the edge is gapped in the non-interacting theory (Fig. 2c).

The electron-electron interactions $\hat{H}_{I}$ are described by

$$
\hat{H}_{I}=\frac{1}{2} \sum_{\sigma, \sigma^{\prime}} \sum_{k, k^{\prime}, q} V_{P}^{\sigma \sigma^{\prime}}\left(k-k^{\prime}, q\right) \hat{\psi}_{k \sigma}^{\dagger} \hat{\psi}_{k^{\prime}, \sigma^{\prime}}^{\dagger} \hat{\psi}_{k^{\prime}+q \sigma^{\prime}} \hat{\psi}_{k-q \sigma},
$$

where $V_{P}^{\sigma \sigma^{\prime}}\left(k-k^{\prime}, q\right)$ is obtained by projecting the Coulomb interactions to the subspace generated by the wavefunctions of the lowest Landau levels (Supplementary Note 2). Here we assumed that the higher Landau levels are energetically separated from the lowest ones by an energy gap larger than the characteristic energy scale of the Coulomb interactions $V_{C}=e^{2} /\left(4 \pi \epsilon \epsilon_{0} l_{B}\right)$. We find that this assumption can be satisfied with the material parameters corresponding to $\mathrm{InAs} / \mathrm{GaSb}$ bilayers $^{25}$.

To find the ground state of the Hamiltonian $\hat{H}=\hat{H}_{0}+\hat{H}_{I}$, we consider states where the local direction of the pseudospin $\mathbf{h}(\mathbf{r})$ $(|\mathbf{h}(\mathbf{r})|=1)$ varies in space. Because the Hamiltonian is translationally invariant in the $x$ direction, we assume that $\mathbf{h}(\mathbf{r})$ is independent of $x$ (It is known that for sufficiently large interlayer separation the quantum Hall bilayers display an instability towards formation of a charge density wave ground state ${ }^{26}$. Here we assume that the interlayer separation is small enough

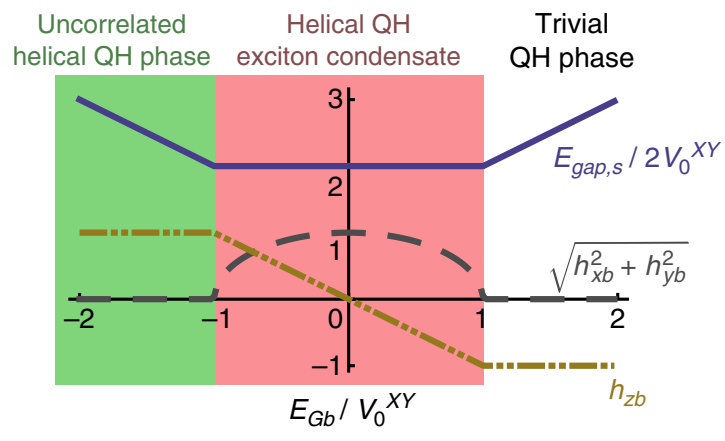

Figure 3 | Phase diagram. For $E_{G b}<-2\left(V_{0}^{X Y}-V_{0}^{Z}\right)$ the system supports an uncorrelated helical $\mathrm{QH}$ phase. In this phase, there is no spontaneous interlayer phase coherence in the bulk $\left(h_{x b}^{2}+h_{y b}^{2}=0\right)$ and the system supports helical edge states (guaranteed by spin-resolved Chern numbers $\left.C_{\uparrow / \downarrow}= \pm 1\right)$. For $\left|E_{G b}\right|<2\left(V_{0}^{X Y}-V_{0}^{Z}\right)$ the system supports a helical QH exciton condensate phase. In this phase, there is spontaneous interlayer phase coherence $\left(h_{x b}^{2}+h_{y b}^{2} \neq 0\right)$, and the system supports exotic confined edge excitations (see below). For $E_{G b}>2\left(V_{0}^{X Y}-V_{0}^{Z}\right)$ the system is in a trivial $\mathrm{QH}$ phase, where the edge is fully gapped. $E_{\mathrm{Gb}}$ can be controlled with gate voltages or magnetic field (If $E_{G b} / V_{0}^{X Y}$ is varied with magnetic field the ratio $V_{0}^{X Y} / V_{0}^{Z}$ will change within the phase-diagram. Nevertheless, we find that the phase-diagram stays qualitatively similar). Charged bulk excitations are gapped everywhere in the phase diagram $\left(E_{\text {gap,s }}>0\right)$. We have chosen $V_{0}^{Z} / V_{0}^{X Y}=1 / 2$.

that such kind of instability does not occur.). By further noticing that the $y$-dependence translates to a momentum dependence of the pseudospin $h_{i}(y)=h_{i}\left(k l_{B}^{2}\right)$, we can express our ansatz for the ground state many-particle wavefunction as a Slater determinant $\left|\Psi\left[\mathbf{h}\left(k l_{B}^{2}\right)\right]\right\rangle$, where for each momentum $k$ we create an electron with pseudospin pointing along $\mathbf{h}\left(k l_{B}^{2}\right)$. To compute the ground state, we need to minimize the energy functional for such kind of pseudospin texture ${ }^{13}$. For the energy functional we obtain (Supplementary Note 2)

$$
\begin{aligned}
E= & E_{0}-\sum_{k, k^{\prime}}\left\{\sum_{i=x, y} V_{P}^{X Y}\left(k-k^{\prime}\right) h_{i}\left(k l_{B}^{2}\right) h_{i}\left(k^{\prime} l_{B}^{2}\right)\right. \\
& \left.+V_{P}^{Z}\left(k-k^{\prime}\right) h_{z}\left(k l_{B}^{2}\right) h_{z}\left(k^{\prime} l_{B}^{2}\right)\right\}+\sum_{k} E_{G}\left(k l_{B}^{2}\right) h_{z}\left(k l_{B}^{2}\right) .
\end{aligned}
$$

Here $\quad V_{P}^{Z}(q)=\left[-V_{P}^{\uparrow \uparrow}(q, 0)+V_{P}^{\uparrow \uparrow}(q, q)+V_{P}^{\uparrow \downarrow}(q, 0)\right] / 4 \quad$ and $V_{P}^{X Y}(q)=V_{P}^{\uparrow \downarrow}(q, q) / 4$ are the interaction coefficients, which characterize the anisotropy of the interactions within a layer and between the layers.

We start by considering an infinite system. In this case, the pseudospin direction $\mathbf{h}(\mathbf{r})$ is spatially homogeneous. By minimizing the energy functional (3), we find that the pseudospin direction $\mathbf{h}_{b}$ is determined by the parameters $E_{\mathrm{Gb}}$ and $V_{0}^{Z(X Y)}=\sum_{q} V_{P}^{Z(X Y)}(q)$ (Supplementary Note 3). Here $E_{\mathrm{Gb}}$ acts as an effective magnetic field preferring the pseudospin direction along $-\operatorname{sgn}\left(E_{G b}\right) \hat{e}_{z}$. On the other hand, the interactions prefer the pseudospin directions within the (x,y)-plane $\left(V_{0}^{X Y}>V_{0}^{Z}\right)$, and the energy cost to rotate the pseudospin so that it points along the $z$ direction is proportional to $V_{0}^{X Y}-V_{0}^{Z}$. Thus, as a balance between these two competing effects, the direction of the pseudospin is tilted away from the $(x, y)$-plane, resulting in the three distinct phases of the system, which are summarized in Fig. 3. For sufficiently large $\left|E_{\mathrm{Gb}}\right|$, we see that $\left|h_{z b}\right|=1$, meaning that only one layer is occupied. The phases $h_{z b}=1$ (uncorrelated helical QH phase) and $h_{z b}=-1$ (trivial $\mathrm{QH}$ phase) are 
Uncorrelated helical QH state (deconfined phase)

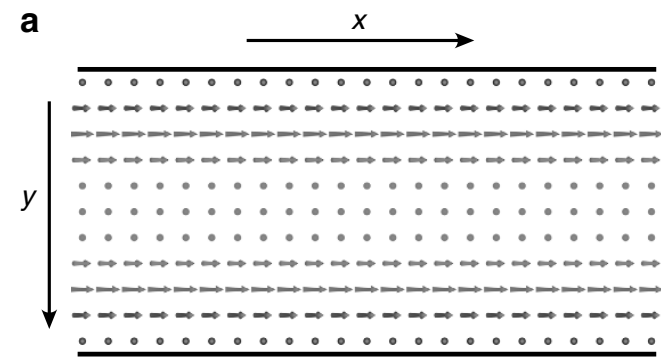

C

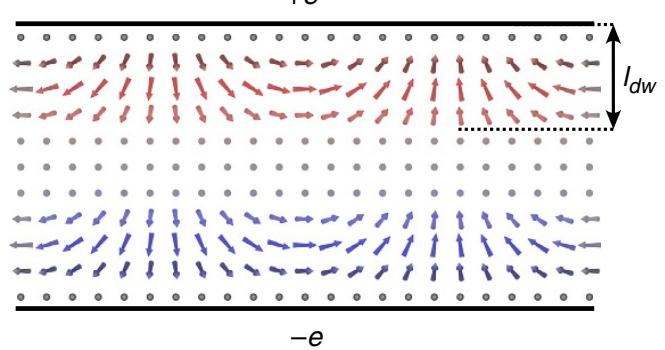

Helical QH exciton condensate (confined phase)

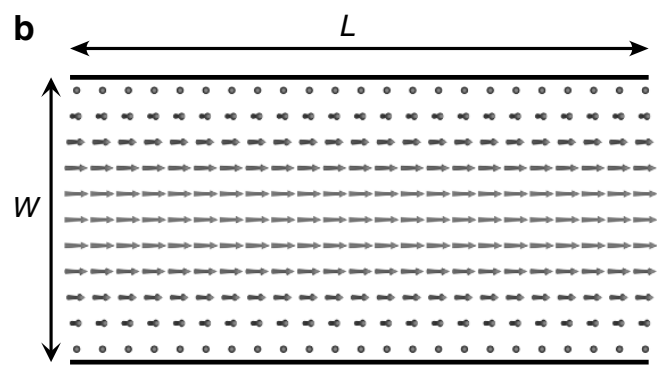

d

$+v \uparrow e$

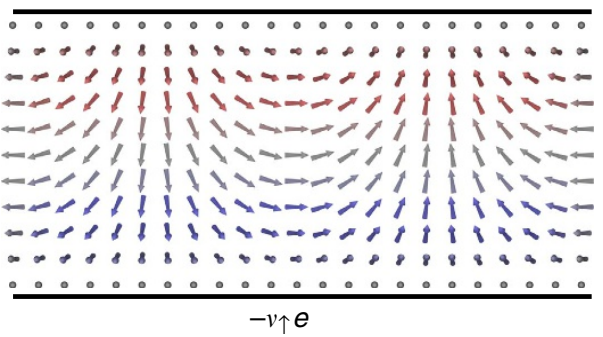

Figure 4 | Spin textures. $(\mathbf{a}, \mathbf{b})$ Ground state spin textures for the uncorrelated helical QH phase and the helical QH exciton condensate phase, respectively. In both cases there exists a robust domain wall, where the polar angle of the pseudospin magnetization $\theta(y)$ rotates from $\pi$ to $\theta_{b}$ along the $y$-direction. In the uncorrelated phase $\theta_{\mathrm{b}}=0$ but in the helical QH exciton condensate phase $\theta_{b} \neq 0, \pi$ indicating spontaneous interlayer phase coherence in the bulk. In both cases the ground states are degenerate for all choices of the constant azimuthal angle $\phi$ of the pseudospin magnetization. (We have chosen $\phi=0$.) $(\mathbf{c}, \mathbf{d})$ Charged excitations can be created by letting the azimuthal angle $\phi(x)$ to rotate along the $x$-direction. In a closed system (obtained by connecting the ends of the sample to form a narrow cylinder) $\phi(x)$ must rotate integer multiples of $2 \pi$. The energy to create such kind of excitation in the uncorrelated helical QH phase scales as $\delta E \sim V_{2}^{X Y} I_{d w} / L$, and the elementary excitations, which carry charge $\pm e$, can be created independently on the different edges. On the other hand, deep inside the helical $\mathrm{QH}$ exciton condensate phase $\delta E \sim V_{2}^{X Y} W / L$ and the elementary charges are $\pm v_{\uparrow} e$. Furthermore, the charged edge excitations are confined: a charge $\pm v_{\uparrow}$ e on one of the edges is always connected to the opposite charge on the other edge by a stripe of rotated pseudospins through the bulk, and thus isolated charges cannot be observed at low energies. The charge density obtained from equation (5) is shown with red (positive charge density) and blue (negative charge density) colours.

topologically distinct from each other. For $E_{G b}<-2\left(V_{0}^{X Y}-V_{0}^{Z}\right)$ and $h_{z b}=1$ the system supports helical edge modes (the spinresolved Chern numbers are $C_{\uparrow / \downarrow}= \pm 1$ ). On the other hand, in the regime $E_{G b}>2\left(V_{0}^{X Y}-V_{0}^{Z}\right)$ and $h_{z b}=-1$, the edge is completely gapped (the spin-resolved Chern numbers are $\left.C_{\uparrow / \downarrow}=0\right)$. Between these two phases is the helical QH exciton condensate phase, where $\left|h_{z b}\right|<1$ and thus $h_{x b}^{2}+h_{y b}^{2} \neq 0$. In this phase the direction of the pseudospin projection onto the $(x, y)$ plane is determined spontaneously. Because the pseudospin in this system labels spin and layer index simultaneously, this phase has simultaneously spontaneous in-plane spin polarization and spontaneous interlayer phase coherence.

The bulk gap for single particle excitations $E_{\text {gap,s }}$ can be calculated using Hartree-Fock linearization (Supplementary Note 3)

$$
E_{\text {gap }, \mathrm{s}}=2 \sqrt{\left(E_{G b}-2 V_{0}^{Z} h_{z b}\right)^{2}+4\left(V_{0}^{X Y}\right)^{2}\left(h_{x b}^{2}+h_{y b}^{2}\right)} .
$$

In addition to the single particle excitations, the helical $\mathrm{QH}$ exciton condensate supports collective excitations ${ }^{13}$ : the neutral pseudospin waves (Goldstone mode) give rise to spin and counterflow charge superfluidity, and the lowest energy charged excitations are topological pseudospin textures, which carry fractional charge $\pm v_{\uparrow(\downarrow)}$ e. Here $v_{\uparrow / \downarrow}=\left(1 \pm h_{z b}\right) / 2$ are the pseudospin resolved filling factors of the different Landau levels. The energy required to create these charged excitations is slightly lower than $E_{\text {gap,s }}$ (ref. 13).

We point out that although $h_{z b}= \pm 1$ are topologically distinct phases, the bulk gap for creating charged excitations never closes, when one tunes from one phase to the other by controlling $E_{\mathrm{Gb}}$. This is possible because the pseudospin rotation symmetry, which protects the existence of spin-resolved Chern numbers as topological numbers, is spontaneously broken in the helical exciton condensate phase. The interacting BHZ model for bilayers shows somewhat similar behaviour also at zero magnetic field, where a trivial insulator phase can be connected to a quantum spin Hall insulator phase without closing of the bulk gap, because of an intermediate phase where the time-reversal symmetry is spontaneously broken ${ }^{27}$. It is also experimentally known that the exciton condensate phase with $v_{\uparrow}=v_{\downarrow}=1 / 2$ can be smoothly connected to uncorrelated $\mathrm{QH}$ state with $v_{\uparrow}=1$ and $v_{\downarrow}=0$ in conventional $\mathrm{QH}$ bilayers $^{28}$. Experimental investigations of InAs/GaSb bilayers in the $\mathrm{QH}$ regime $^{18,29}$ are consistent with this prediction, because no gap closing has been observed as a function of magnetic field.

Confinement-deconfinement transition of edge excitations. We now turn to the description of the ground state pseudospin texture $h_{z}(y)=\cos \left[\theta_{0}(y)\right], h_{x}(y)=\sin \left[\theta_{0}(y)\right] \cos (\phi)$ and $h_{y}(y)=$ $\sin \left[\theta_{0}(y)\right] \sin (\phi)$ at the edge. (The ground state will be degenerate with respect to the choice of $\phi$.) As discussed above close to the edge $E_{G}(y)>0$ takes large values, because the edge states are topologically protected to exist at all energies between the lowest Landau levels and higher ones. Therefore close to the edge $\theta_{0}(y)=\pi$. On the other hand, in the bulk $\theta_{0}(y)=\theta_{b}=\arccos \left(h_{z b}\right)$. This means that there always exists a domain wall, where $\theta_{0}$ rotates from $\pi$ to $\theta_{b}$. Although the existence of the domain wall is a robust topological property of the system, the detailed shape of 
$\theta_{0}(y)$ and the length scale $l_{d w}$, where this rotation happens, depend on the details of the sample (Supplementary Note 4$)$. The ground states in the uncorrelated helical $\mathrm{QH}$ phase $\left(\theta_{b}=0\right)$ and helical QH exciton condensate phase $\left(\theta_{b} \neq 0, \pi\right)$ are illustrated in Fig. 4a,b, respectively. It turns out that the existence of spontaneous interlayer phase coherence, which distinguishes the two different phases of matter, also has deep consequences on the nature of the low-energy excitations in this system.

By using a Hartree-Fock linearization for the ground state, we find that the single particle excitations are gapped also close to the edge, and the magnitude of the energy gap is determined by the Coulomb energy scale $V_{0}^{X Y}$. However, similarly as for the case of a coherent domain wall in $\mathrm{QH}$ ferromagnetic state in graphene ${ }^{23}$, the lowest energy edge excitations are not the single-particle ones. Namely, the ground state is degenerate with respect to the choice of $\phi$, and therefore in accordance with the Goldstone's theorem the system supports low-energy excitations described by spatial variation of $\phi(\mathbf{r})$. Due to the general relationship between the electric and topological charge densities in $\mathrm{QH}$ ferromagnets ${ }^{13,23}$ (Supplementary Note 5)

$$
\delta \rho(\mathbf{r})=-\frac{e}{4 \pi} \frac{\partial \phi}{\partial x} \frac{\partial \cos \left[\theta_{0}(y)\right]}{\partial y}
$$

these excitations also carry charge, which is localized at the edges of the sample. In this section we illustrate these excitations in a closed system obtained by connecting the ends of the sample to form a narrow cylinder with width $W$ and circumference $L$ (Alternatively, instead of comparing the energies to create elementary excitations in a closed system one could compare the energies needed to create a fixed charge density on the edge. This generalization allows the possibility to consider open systems.). We point out that in addition to the topological contribution (5) there can also be non-topological contributions to the electric charge density of pseudospin textures due to excitation of higher Landau levels. However, these contributions

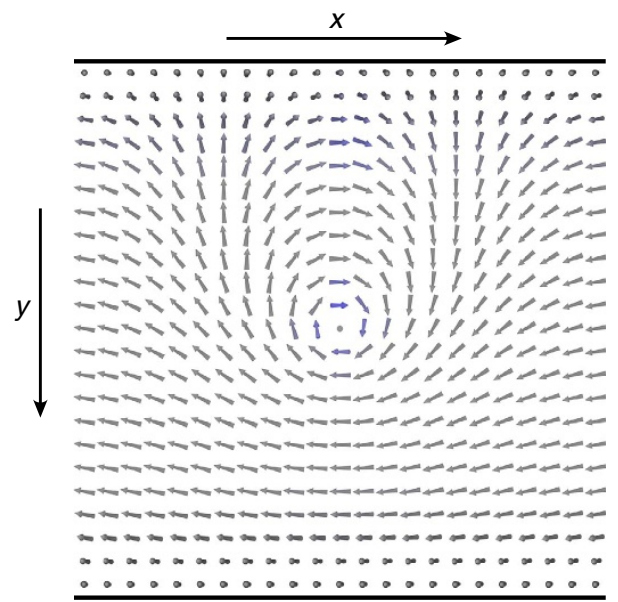

Figure 5 | Charged excitation localized at one edge of the sample in the helical QH exciton condensate. The excitation can be visualized as a meron-antimeron pair, where the meron is localized inside the sample and anti-meron-outside. These excitations become the lowest energy charged excitations in sufficiently wide samples (otherwise similar cylinder geometry as in Fig. 4), where it is energetically favourable to break the stripe connecting opposite charges at the different edges by a creation of a bulk meron. Depending on the profile $E_{G}(y)$ near the edge, the lowest energy excitation is charge-neutral or having charge $\pm e$. The charge is determined by the pseudospin orientation in the center of the meron. The energy of such excitation is of order of Coulomb energy $V_{0}^{X Y}$. are small for the pseudospin textures which are smooth on the scale of $l_{B}$ (Supplementary Note 8 and Supplementary Fig. 2).

We start by considering this kind of closed system, where $W<<L$ (see Fig. 4). This geometry is topologically equivalent to a Corbino ring, which has been experimentally realized for $\mathrm{QH}$ exciton condensates ${ }^{30,31}$. Using equations (3) and (5) we find that the lowest energy excitations correspond to rotation of $\phi(x)$ by $2 \pi$ and carry a net charge within one of the edges (Supplementary Note 6). They have an energy $\delta E \sim V_{2}^{X Y} l_{d w} / L$ in the uncorrelated phase and $\delta E \sim V_{2}^{X Y} W / L$ deep in the helical exciton condensate phase. Here $V_{2}^{X Y}=\frac{1}{2} \sum_{q} V_{P}^{X Y}(q) q^{2} l_{B}^{2}$ characterizes the cost of exchange energy caused by $\nabla \phi(x)$ (Supplementary Note 4 ).

In the uncorrelated phase these excitations have a charge $\pm e$. By inspecting Fig. 4, we notice that because the spin points along $z$-direction in the bulk, there is no rotation happening in the bulk. This means that we can choose separate fields $\phi_{1}(x)$ and $\phi_{2}(x)$ for the two edges, so that these excitations can be created independently on the different edges much as in graphene ${ }^{23}$.

The situation is dramatically different in the helical QH exciton condensate phase. There, the elementary excitations in a closed system have a charge $\pm v_{\uparrow}$. Moreover, as illustrated in Fig. 4, a charge $\pm v_{\uparrow} e$ on one of the edges is always connected to the opposite charge on the other edge by a stripe of rotated bulk pseudospins. Breaking the bulk pseudospin configuration costs an energy comparable to the Coulomb energy, and thus isolated charges cannot be observed at low energies. This means that this type of charged edge excitations in the helical $\mathrm{QH}$ exciton condensate phase are confined.

It is illustrative to consider what happens to the excitations in the helical QH exciton condensate, when the width of the sample is increased. Namely, the excitation energy increases proportionally to the width of the sample $\delta E \sim V_{2}^{X Y} W / L$ and eventually for $W \sim L$ it becomes energetically favourable instead of having a
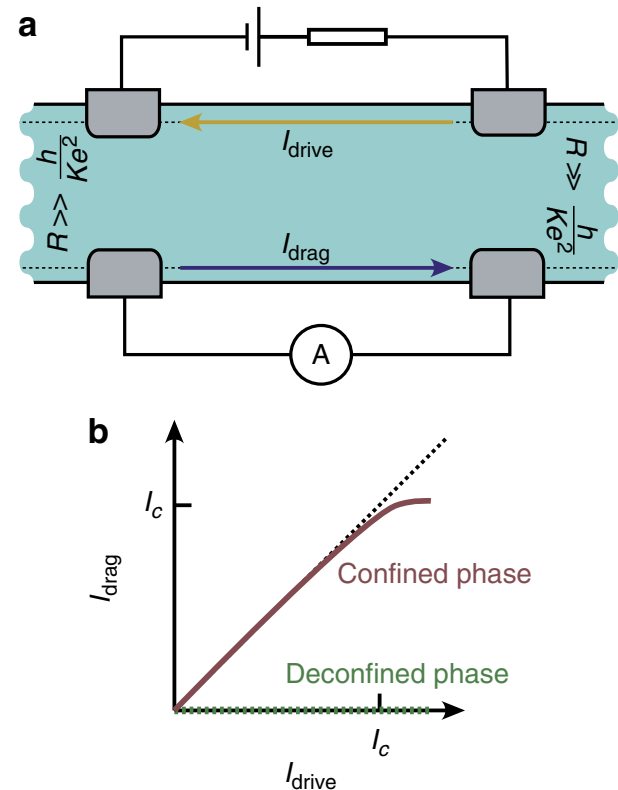

Figure 6 | Non-local transport to demonstrate the charge confinement. (a) The transport geometry. A drive current is applied on one of the edges and the resulting drag current is measured on the opposite edge. (b) Drag current $I_{\text {drag }}$ as a function of the driving current $I_{\text {drive }}$ in the geometry from a. Due to the charge confinement in the helical QH exciton condensate phase, the drive current necessarily gives rises to an opposite drag current on the other edge. On the other hand, in the uncorrelated (deconfined) phase, there is only a weak drag current due to the Coulomb force acting between the charges. 
large area of rotating spins between two edges to create a bulk meron (Fig. 5). This resembles the physics of quarks, where the growing separation of a quark-antiquark pair eventually results in the creation of a new quark-antiquark pair between them.

Luttinger liquid theory and nonlocal transport. To predict experimentally measurable consequences of the charge confinement, we consider nonlocal transport in an open system. By considering the time-dependent field theory for the pseudospin for a reasonably narrow sample in the helical $\mathrm{QH}$ exciton condensate phase we arrive at an effective one-dimensional Hamiltonian (Supplementary Note 7)

$$
H=\int d x\left[\frac{e^{2}}{2 W \hbar^{2} \Gamma} \Pi(x)^{2}+\frac{W \rho_{s b}}{2}\left(\partial_{x} \phi(x)\right)^{2}\right],
$$

where $\rho_{s b}=V_{2}^{X Y} \sin ^{2} \theta_{b} / \pi$ is the pseudospin stiffness and $\Gamma=e^{2} /\left(16 \pi l_{B}^{2}\left(V_{0}^{X Y}-V_{0}^{Z}\right)\right)$ describes the interlayer capacitance per unit area, which is strongly enhanced from the electrostatic value by the exchange interactions. The one-dimensional charge densities in the different edges (labelled 1 and 2) $\rho_{1,2}(x)=\mp \frac{e}{4 \pi}\left(1+\cos \theta_{b}\right) \frac{\partial \phi}{\partial x}$ are always opposite and determined by a single field $\phi(x)$, highlighting the confinement of the charged edge excitations. The one-dimensional theory describes a Luttinger liquid, and the so-called Luttinger parameter $K$ in the convention used in ref. 32 , is given by

$$
K=\frac{l_{B}}{W} \sqrt{\frac{V_{0}^{X Y}-V_{0}^{Z}}{V_{2}^{X Y}}} \frac{\left(1+\cos \theta_{b}\right)^{2}}{4 \sin \theta_{b}} .
$$

The Luttinger parameter in quantum Hall systems determines the conductance for ideal contacts $G_{c f}=K e^{2} / h$ (ref. 32,33). Because the pseudospin waves are charge neutral in the bulk, conductance decreases with $W$ as $G_{c f} \propto 1 / W$. It is important to notice that in this system $G_{c f}$ describes the conductance for a counterflow/drag geometry, where opposite currents are flowing in the two edges. The helical QH exciton condensate phase does not support net transport current as long as the voltages $e V$ are small compared with $\hbar v \pi / W$ (Supplementary Note 7 ). This automatically leads to a remarkable transport property that characterizes the helical $\mathrm{QH}$ exciton condensate phase. Namely, by considering a non-local transport geometry shown in Fig. 6a, where a drive current is applied on one of the edges and a resulting drag current is measured on the opposite edge, we find that necessarily $I_{\text {drag }}=I_{\text {drive }}$ at small voltages. This should be contrasted to the uncorrelated helical phase, where the charged edge excitations are deconfined. In that case, one has two independent Luttinger liquid theories for the two edges (Supplementary Note 7), and therefore one expects only a weak drag current due to the Coulomb force acting between the charges. For $W \gg l_{B}$, we expect that this effect is negligible compared with drag current in the confined phase.

Finally, to estimate the critical current $I_{c}$, where the relation $I_{\text {drag }}=I_{\text {drive }}$ breaks down, we notice that the maximum voltage is determined by the gap $e V_{\max } \approx \hbar v \pi / W$. By using reasonable estimates $V_{0}^{Z}=V_{0}^{X Y} / 2, \quad V_{2}^{X Y}=V_{0}^{X Y} / 4, \quad \theta_{b}=\pi / 2$, $W=20 \quad l_{B}, \quad l_{B}=10 \mathrm{~nm}, \quad v=14 \mathrm{~km} \mathrm{~s}^{-1}$ (refs 34,35), we find $I_{c}=G_{c f} V_{\text {max }} \approx 0.1 \mathrm{nA}$.

\section{Discussion}

In summary, we have predicted the existence of a helical $\mathrm{QH}$ exciton condensate state in band-inverted electron-hole bilayers. We have shown that the counterpropagating edge modes give rise to a ground state pseudospin texture, where the polar angle of the pseudospin magnetization $\theta(y)$ rotates from the boundary value $\pi$ to the bulk value $\theta_{b}$ along the direction perpendicular to the edge.
Low-energy charged excitations can be created by letting the azimuthal angle of the pseudospin polarization $\phi(x)$ to rotate along the edge. Remarkably, in a sufficiently narrow Hall bar these charged edge excitations are confined in the presence of spontaneous interlayer phase-coherence $\left(\theta_{b} \neq 0, \pi\right)$ : a charge on one of the edges always gives rise to the opposite charge on the other edge, and thus isolated charges cannot be observed at low energies. Moreover, we predict the possibility to control $\theta_{b}$ with a magnetic field and gate voltages. This allows to study a confinement-deconfinement transition, which occurs simultaneously with the bulk phase-transition between the helical $\mathrm{QH}$ exciton condensate phase $\left(\theta_{b} \neq 0, \pi\right)$ and the uncorrelated helical QH phase $\left(\theta_{b}=0\right)$.

The helical QH exciton condensate phase can be experimentally probed using Josephson-like interlayer tunnelling and counterflow superfluidity 1,8,9,13,30,31,34,35. Moreover, because the pseudospin in this system describes simultaneously both the spin and the layer degrees of freedom, the helical QH exciton condensate phase can also be probed using the spin superfluidity and the NMR techniques ${ }^{8}$. Perhaps it is even possible to use local probe techniques to image the confinementdeconfinement transition and the confinement physics as illustrated in Figs 4 and 5. Finally, we have shown that the charge confinement also gives rise to a remarkable new transport property. Namely, a drive current applied on one of the edges gives rise to exactly opposite drag current $I_{\mathrm{drag}}=I_{\text {drive }}$ at the other edge.

Our results for the confinement of the edge excitations may also be applicable to the so-called canted antiferromagnetic phase, which is predicted to appear in graphene ${ }^{36}$. Similarly to the helical QH exciton condensate state considered in this paper, the canted antiferromagnetic phase is characterized by a spontaneously broken U(1)-symmetry in the bulk and a single edge supports only gapped meron-antimeron excitations ${ }^{36}$.

The phenomena of confinement stemming from the particle physics models ${ }^{24}$ has been studied also in condensed matter systems ${ }^{37-41}$. However, we expect that the combination of the different techniques for probing the helical $\mathrm{QH}$ exciton condensate phase will provide a more intuitive understanding and new perspectives on the confinement physics.

We also point out that InAs/GaSb bilayers is a promising system for superconducting applications, and edge-mode superconductivity has already been experimentally demonstrated in the QSH regime ${ }^{42}$. In the presence of superconducting contacts, the helical QH exciton condensate may provide a new route for realizing exotic non-local Josephson effects and non-Abelian excitations, such as parafermions ${ }^{5,6}$.

\section{References}

1. Sarma, S. D. \& Pinczuk, A. Perspectives in quantum hall effects: Novel quantum liquids in low-dimensional semiconductor structures (John Wiley \& Sons, 2008).

2. Jain, J. Composite Fermions (Cambridge University Press, 2007).

3. Read, N. \& Green, D. Paired states of fermions in two dimensions with breaking of parity and time-reversal symmetries and the fractional quantum Hall effect. Phys. Rev. B 61, 10267 (2000).

4. Nayak, C., Simon, S. H., Stern, A., Freedman, M. \& Sarma, S. D. Non-Abelian anyons and topological quantum computation. Rev. Mod. Phys. 80, 1083 (2008).

5. Lindner, N. H., Berg, E., Refael, G. \& Stern, A. Frac-tionalizing Majorana fermions: non-abelian statistics on the edges of abelian quantum Hall states. Phys. Rev. X 2, 041002 (2012).

6. Clarke, D. J., Alicea, J. \& Shtengel, K. Exotic non-Abelian anyons from conventional fractional quantum Hall states. Nat. Commun. 4, 1348 (2013).

7. Mong, R. S. et al. Universal topological quantum computation from a superconductor-abelian quantum Hall heterostructure. Phys. Rev. X 4, 011036 (2014).

8. Girvin, S. The Quantum Hall Effect: Novel Excitations and Broken Symmetries (Indiana University, 1999). 
9. Eisenstein, J. \& MacDonald, A. Bose-Einstein condensation of excitons in bilayer electron systems. Nature 432, 691-694 (2004).

10. Chakraborty, T. \& Pietilainen, P. Fractional quantum Hall effect at half-filled landau level in a multiple-layer electron system. Phys. Rev. Lett. 59, 2784 (1987).

11. Fertig, H. Energy spectrum of a layered system in a strong magnetic field. Phys. Rev. B 40, 1087 (1989).

12. Sondhi, S., Karlhede, A., Kivelson, S. \& Rezayi, E. Skyrmions and the crossover from the integer to fractional quantum Hall effect at small Zeeman energies. Phys. Rev. B 47, 16419 (1993).

13. Moon, K. et al. Spontaneous interlayer coherence in double-layer quantum Hall systems: charged vortices and Kosterlitz-Thouless phase transitions. Phys. Rev. B 51, 5138 (1995).

14. Kane, C. L. \& Mele, E. J. $\mathrm{L}_{2}$ topological order and the quantum spin Hall effect. Phys. Rev. Lett. 95, 146802 (2005).

15. Bernevig, B. A., Hughes, T. L. \& Zhang, S.-C. Quantum spin hall effect and topological phase transition in HgTe quantum wells. Science 314, 1757-1761 (2006).

16. Kaonig, M. et al. Quantum spin hall insulator state in $\mathrm{HgTe}$ quantum wells. Science 318, 766-770 (2007).

17. Liu, C., Hughes, T. L., Qi, X.-L., Wang, K. \& Zhang, S.-C. Quantum spin hall effect in inverted Type-II semiconductors. Phys. Rev. Lett. 100, 236601 (2008).

18. Du, L., Knez, I., Sullivan, G. \& Du, R.-R. Robust helical edge transport in gated InAs/GaSb bilayers. Phys. Rev. Lett. 114, 096802 (2015).

19. Spanton, E. M. et al. Images of edge current in InAs/GaSb quantum wells. Phys. Rev. Lett. 113, 026804 (2014)

20. Scharf, B., Matos-Abiague, A. \& Fabian, J. Magnetic properties of HgTe quantum wells. Phys. Rev. B 86, 075418 (2012).

21. Pikulin, D. I. et al. Disorder and magnetic-field-induced breakdown of helical edge conduction in an inverted electron-hole bilayer. Phys. Rev. B 89, 161403 (2014).

22. Fal'ko, V. \& Iordanskii, S. V. Topological defects and goldstone excitations in domain walls between ferromagnetic quantum hall liquids. Phys. Rev. Lett. 82, 402 (1999).

23. Fertig, H. A. \& Brey, L. Luttinger liquid at the edge of undoped graphene in a strong magnetic field. Phys. Rev. Lett 97, 116805 (2006).

24. Greensite, J. An Introduction to the Confinement Problem (Springer, 2011)

25. Liu, C. \& Zhang, S.-C. in Topological Insulators (eds Franz, M. \& Molenkamp, L. W.) (Elsevier, 2013).

26. Cote, R., Brey, L. \& MacDonald, A. H. Broken-symmetry ground states for the two-dimensional electron gas in a double-quantum-well system. Phys. Rev. B 46, 10239 (1992).

27. Pikulin, D. \& Hyart, T. Interplay of exciton condensation and the quantum spin Hall effect in InAs/GaSb Bilayers. Phys. Rev. Lett. 112, 176403 (2014).

28. Zhang, D. et al. Local compressibility measurement of the $\mathrm{v}_{\text {tot }}=1$ quantum Hall state in a bilayer electron system. Phys. Rev. B 87, 205304 (2013).

29. Nichele, F. et al. Insulating state and giant nonlocal response in an InAs/GaSb quantum well in the quantum hall regime. Phys. Rev. Lett. 112, 036802 (2014).

30. Finck, A. D. K., Eisenstein, J. P., Pfeiffer, L. N. \& West, K. W. Exciton transport and andreev reflection in a bilayer quantum Hall system. Phys. Rev. Lett. 106, 236807 (2011).

31. Huang, X., Dietsche, W., Hauser, M. \& von Klitz-ing, K. Coupling of Josephson Currents in Quantum Hall Bilayers. Phys. Rev. Lett. 109, 156802 (2012).

32. Giamarchi, T. Quantum Physics in One Dimension (Oxford University Press, 2003).
33. Kane, C. L. \& Fisher, M. P. A. Contacts and Edge State Equilibration in the Fractional Quantum Hall Effect. Phys. Rev. B 52, 17393 (1995).

34. Spielman, I. B., Eisenstein, J. P., Pfeiffer, L. N. \& West, K. W. Observation of a linearly dispersing collective mode in a quantum Hall ferromagnet. Phys. Rev. Lett. 87, 036803 (2001).

35. Hyart, T. \& Rosenow, B. Quantitative description of Josephson-like tunneling in $\mathrm{v}_{\mathrm{T}}=1$ quantum Hall bilayers. Phys. Rev. B 83, 155315 (2011).

36. Murthy, G., Shimshoni, E. \& Fertig, H. A. Collective edge modes near the onset of a graphene quantum spin Hall state. Phys. Rev. B 90, 241410(R) (2014).

37. Volovik, G. E. The Universe in a Helium Droplet (Clarendon Press, 2003).

38. Volovik, G. E. Monopoles and fractional vortices in chiral superconductors. Proc. Natl Acad. Sci. USA 97, 2431-2436 (2000).

39. Kondo, Y. et al. Combined spin-mass vortex with soliton tail in superfluid ${ }^{3} \mathrm{~B}$ Phys. Rev. Lett. 68, 3331 (1992).

40. Yang, K. et al. Spontaneous interlayer coherence in double-layer quantum Hall systems: symmetry-breaking interactions, in-plane fields, and phase soliton. Phys. Rev. B 54, 11644 (1996).

41. Lake, B. et al. Confinement of fractional quantum number particles in a condensed-matter system. Nat. Phys. 6, 50-55 (2010).

42. Pribiag, V. S. et al. Edge-mode superconductivity in a two-dimensional topo-logical insulator. Nat. Nanotechnol. 10, 593-597 (2015).

\section{Acknowledgements}

This work was supported by the Academy of Finland Center of Excellence program, the European Research Council (Grant No. 240362-Heattronics), the Dutch Science Foundation NWO/FOM, NSERC, CIfAR, Max Planck-UBC Centre for Quantum Materials, and the DFG grant RE 2978/1-1.

\section{Author contributions}

All authors contributed at all stages of the project: generating ideas for the project, making calculations and writing the manuscript

\section{Additional information}

Supplementary Information accompanies this paper at http://www.nature.com/ naturecommunications

Competing financial interests: The authors declare no competing financial interests.

Reprints and permission information is available online at http://npg.nature.com/ reprintsandpermissions/

How to cite this article: Pikulin, D. I. et al. Confinement-deconfinement transition due to spontaneous symmetry breaking in quantum Hall bilayers. Nat. Commun. 7:10462 doi: $10.1038 /$ ncomms10462 (2016).

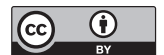

This work is licensed under a Creative Commons Attribution 4.0 International License. The images or other third party material in this article are included in the article's Creative Commons license, unless indicated otherwise in the credit line; if the material is not included under the Creative Commons license, users will need to obtain permission from the license holder to reproduce the material To view a copy of this license, visit http://creativecommons.org/licenses/by/4.0/ 